\title{
The Banach-Saks Properties in Orlicz-Lorentz Spaces
}

\author{
Anna Kamińska ${ }^{1}$ and Han Ju Lee ${ }^{2}$ \\ ${ }^{1}$ Department of Mathematical Sciences, The University of Memphis, Memphis, TN 38152, USA \\ ${ }^{2}$ Department of Mathematics Education, Dongguk University-Seoul, Seoul 100-715, Republic of Korea
}

Correspondence should be addressed to Han Ju Lee; hanjulee@dongguk.edu

Received 30 November 2013; Accepted 7 February 2014; Published 13 March 2014

Academic Editor: Mieczysław Mastyło

Copyright (c) 2014 A. Kamińska and H. J. Lee. This is an open access article distributed under the Creative Commons Attribution License, which permits unrestricted use, distribution, and reproduction in any medium, provided the original work is properly cited.

The Banach-Saks index of an Orlicz-Lorentz space $\Lambda_{\varphi, w}(I)$ for both function and sequence case, is computed with respect to its Matuszewska-Orlicz indices of $\varphi$. It is also shown that an Orlicz-Lorentz function space has weak Banach-Saks (resp., Banach-Saks) property if and only if it is separable (resp., reflexive).

\section{Introduction}

Let $(X,\|\cdot\|)$ be a real Banach space. A bounded sequence $\left\{x_{n}\right\} \subset X$ is called a weak Banach-Saks sequence whenever there exists a subsequence $\left\{y_{n}\right\} \subset\left\{x_{n}\right\}$ such that its Cesàro means converge in norm to zero; that is,

$$
\frac{1}{n} \sum_{i=1}^{n} y_{i} \longrightarrow 0
$$

The space $X$ is said to satisfy the weak Banach-Saks property, in short $X \in(w B S)$, if every weakly null sequence $\left\{x_{n}\right\}$ in $X$ is a weak Banach-Saks sequence. Recall that a Banach space $X$ satisfies the Banach-Saks property $(B S)$ if for every bounded sequence $\left\{x_{n}\right\}$ in $X$, there is a subsequence $\left\{y_{j}\right\}$ such that its Cesàro means converge; that is, the sequence $\left\{(1 / m) \sum_{j=1}^{m} y_{j}\right\}$ is convergent in norm. It is well known that a Banach space has the (BS)-property if and only if it is reflexive and it has the $(w B S)$-property [1].

Schreier constructed a separable Banach space without the weak Banach-Saks property [2] (which is called a Schreier space) and later it was shown by Baernstein [3] that there is a reflexive separable Banach space without the weak Banach-Saks property. Hence the space $\ell_{\infty}$ does not have the weak Banach-Saks property, since every separable space is embeddable in $\ell_{\infty}$.
Following Johnson [4], given $1 \leq p \leq \infty$, a bounded sequence $\left\{x_{n}\right\} \subset X$ is called a $B S_{p}$ sequence if there exists a subsequence $\left\{y_{n}\right\} \subset\left\{x_{n}\right\}$ such that

$$
\sup _{m \in \mathbb{N}} m^{-1 / p}\left\|\sum_{i=1}^{m} y_{i}\right\|<\infty .
$$

Here $m^{1 / \infty}=1$ for all $m \in \mathbb{N}$. We then say that $X$ has the $p$ Banach-Saks property, shortly $X \in\left(B S_{p}\right)$, if each weakly null sequence contains a $B S_{p}$ subsequence. It is clear that every Banach space has a $\left(B S_{1}\right)$ property. The Banach-Saks index is the number $\gamma(X)=\sup \left\{p: X \in\left(B S_{p}\right)\right\}$. The set $\Gamma(X)=$ $\left\{p: X \in\left(B S_{p}\right)\right\}$ is always an interval $[1, \gamma(X))$ or $[1, \gamma(X)]$. The Bessaga-Pełczyński method [5, Proposition 1.a.12] shows that $\Gamma\left(\ell_{p}\right)=[1, p]$ and the Schur property of $\ell_{1}$ shows that $\Gamma\left(\ell_{1}\right)=[1, \infty]$. The Banach-Saks index of Nakano sequence space was computed in [6].

The stronger property $\left(S_{p}\right)$ was introduced by Knaust and Odell in [7]. It is said that $X$ has property $\left(S_{p}\right), 1<p \leq \infty$, if every weakly null sequence in $X$ has a subsequence $\left\{x_{k}\right\}$ so that there is a constant $C>0$ such that, for all $m \in \mathbb{N}$ and all real sequences $a=\left(a_{n}\right) \in \ell_{p}$,

$$
\left\|\sum_{j=1}^{m} a_{j} x_{j}\right\| \leq C\|a\|_{p},
$$

where $\|a\|_{p}$ is the $\ell_{p}$ norm. 
It is clear that $\left(S_{p}\right) \Rightarrow\left(B S_{p}\right) \Rightarrow(w B S)$ for all $1<$ $p \leq \infty$. The Elton $c_{0}$-theorem [8, Theorem III 3.5] states that $\left(B S_{\infty}\right) \Leftrightarrow\left(S_{\infty}\right)$ and a Banach space $X$ has $\left(B S_{\infty}\right)$ if and only if every normalized weakly null sequence contains a subsequence which is equivalent to the unit vector basis of $c_{0}$, which is equivalent to the case in which every subspace of $X$ has the Dunford-Pettis property (for details see [9]). In general, the two properties $\left(B S_{p}\right)$ and $\left(S_{p}\right)$ are not equivalent if $1<p<\infty$ [10]; however, Rakov [11, Theorem 3] showed that if $1<q<p<\infty$, then $\left(B S_{p}\right)$ implies $\left(S_{q}\right)$. Various weak Banach-Saks properties and their relations were shown in [6] for Musielak-Orlicz sequence spaces.

In this paper we compute the Banach-Saks index of Orlicz-Lorentz space $\Lambda_{\varphi, w}(I)$ and show that Orlicz-Lorentz function space $\Lambda_{\varphi, w}(I)$ has the weak Banach-Saks property (resp., Banach-Saks property) if and only if it is separable (resp., reflexive). We start with several preliminary results about Orlicz-Lorentz spaces.

\section{Preliminaries}

Let $I=\mathbb{N}$ or $I=(0, a)$ with $0<a \leq \infty$, equipped with the counting measure if $I=\mathbb{N}$ and the Lebesgue measure if $I=(0, a)$. If $A \subset I$ is a measurable set, we denote by $|A|$ its measure. By $L^{0}(I)$ we denote the collection of all real valued measurable functions on $I$. In the case when $I=\mathbb{N}$ the elements are sequences $x=(x(n))$, and in the other cases they are real valued Lebesgue measurable functions $x$.

For a measurable function $x$, the decreasing rearrangement of $x$ will be denoted by $x^{*}$, defined as $x^{*}(t)=\inf \{s>$ $\left.0: d_{|x|}(s) \leq t\right\}, t \geq 0$, where $d_{x}(s)=|\{t: x(t) \geq s\}|, s \geq 0$. For two measurable functions $x$ and $y$, the relation $x \prec \prec y$ means that, for all $t \geq 0$, we have

$$
\int_{0}^{t} x^{*}(s) d s \leq \int_{0}^{t} y^{*}(s) d s
$$

For a sequence $x=(x(n)), x^{*}$ will denote the decreasing rearrangement of $x$. That is, letting $\tilde{x}(t)=\sum_{n=1}^{\infty} x(n) \chi_{[n-1,1)}$, we have $x^{*}(n)=\tilde{x}^{*}(n-1)$ for all $n \in \mathbb{N}$. For sequences $x$ and $y, x \prec \prec y$ means that for all $n \in \mathbb{N}$, we have

$$
\sum_{k=1}^{n} x^{*}(k) \leq \sum_{k=1}^{n} y^{*}(k) .
$$

Let $\varphi: \mathbb{R}_{+} \rightarrow \mathbb{R}_{+}$be an Orlicz function; that is, $\varphi$ is convex, strictly increasing, and $\varphi(0)=0$. Let the weight function $w \in L^{0}(I)$ be nonnegative and nonincreasing. For $I=(0, a), 0<a \leq \infty$, the Orlicz-Lorentz space $\Lambda_{\varphi, w}(I)=$ $\Lambda_{\varphi, w}(0, a)$ is the collection of all functions $f \in L^{0}(0, a)$ such that

$$
\|f\|=\|f\|_{\varphi, w}=\inf \left\{\epsilon>0: I\left(\frac{f}{\epsilon}\right) \leq 1\right\}<\infty,
$$

where $I(f)=I_{\varphi, w}(f)=\int_{I} \varphi\left(f^{*}\right) w$. Analogously in case when $I=\mathbb{N}$, the Orlicz-Lorentz sequence space $d(\varphi, w)=\Lambda_{\varphi, w}(\mathbb{N})$ contains all real sequences $x=(x(n))$ such that

$$
\|x\|=\|x\|_{\varphi, w}=\inf \left\{\epsilon>0: I\left(\frac{x}{\epsilon}\right) \leq 1\right\}<\infty
$$

where $I(x)=I_{\varphi, w}(x)=\sum_{n=1}^{\infty} \varphi\left(x^{*}(n)\right) w(n)$. It is well known that the space $\left(\Lambda_{\varphi, w}(I),\|\cdot\|\right)$ is a rearrangement invariant (r.i.) Banach function space satisfying the Fatou property [12-15]. The space $\Lambda_{\varphi, w}^{0}(0, a)$ (resp., $\left.d^{0}(\varphi, w)\right)$ is the order continuous part of $\Lambda_{\varphi, w}(0, a)$ (resp., $\left.d(\varphi, w)\right)$. In the sequence case $d^{0}(\varphi, w)$ is the closure of the linear span of the unit vectors $\left(e_{i}\right)$ in $d(\varphi, w)$. If $1 \leq p<\infty$ and $\varphi(u)=u^{p}$, then $d(\varphi, w)=d(p, w)$ and $\Lambda_{\varphi, w}(0, a)=\Lambda_{p, w}(0, a)[16]$. If $w \equiv 1$, then $\Lambda_{\varphi, w}(0, a)=L_{\varphi}(0, a)$ is an Orlicz function space, and $d(\varphi, w)=\ell_{\varphi}$ is an Orlicz sequence space. In this case also $\Lambda_{\varphi, w}^{0}(0, a)=L_{\varphi}^{0}(0, a)$ and $d^{0}(\varphi, w)=\ell_{\varphi}^{0}$ are subspaces of order continuous elements in Orlicz spaces.

We may assume that $\varphi(1)=1$. In fact, the space $\Lambda_{\psi, w}(I)$, where $\psi(t)=\varphi(\alpha t)$ with $\varphi(\alpha)=1$, is isometric to $\Lambda_{\varphi, w}(I)$; we have $\|f\|_{\psi, w}=\alpha\|f\|_{\varphi, w}$.

Definition 1. Given an Orlicz function $\varphi$, one defines the growth conditions connected to its lower and upper Matuszewska-Orlicz indices in three different categories, at zero, at infinity, and on $\mathbb{R}_{+}$.

We say that, for $1 \leq p<\infty, \varphi \in \Delta_{0}^{* p}, \varphi \in \Delta_{\infty}^{* p}$, and $\varphi \in \Delta^{* p}$, whenever

$$
\begin{aligned}
& \sup _{\substack{0<a \leq 1 \\
0<t<1}} \frac{\varphi(a t)}{\varphi(t) a^{p}}<\infty, \quad \sup _{\substack{0<a \leq 1 \\
t>1}} \frac{\varphi(a t)}{\varphi(t) a^{p}}<\infty, \\
& \sup _{\substack{0<a \leq 1 \\
t>0}} \frac{\varphi(a t)}{\varphi(t) a^{p}}<\infty,
\end{aligned}
$$

respectively. Analogously we say that, given $1 \leq q<\infty, \varphi \in$ $\Delta_{0}^{q}, \varphi \in \Delta_{\infty}^{q}$, and $\varphi \in \Delta^{q}$, whenever

$$
\begin{gathered}
\inf _{\substack{0<a \leq 1 \\
0<t<1}} \frac{\varphi(a t)}{\varphi(t) a^{q}}>0, \quad \inf _{\substack{0<a \leq 1 \\
t>1}} \frac{\varphi(a t)}{\varphi(t) a^{q}}>0, \\
\inf _{\substack{0<a \leq 1 \\
t>0}} \frac{\varphi(a t)}{\varphi(t) a^{q}}>0,
\end{gathered}
$$

respectively.

The Matuszewska-Orlicz indices, lower $\alpha_{\varphi}^{0}, \alpha_{\varphi}^{\infty}$, and $\alpha_{\varphi}$ and upper $\beta_{\varphi}^{0}, \beta_{\varphi}^{\infty}$, and $\beta_{\varphi}$, are then defined as follows:

$$
\begin{gathered}
\alpha_{\varphi}^{0}=\sup \left\{p: \varphi \in \Delta_{0}^{* p}\right\}, \quad \alpha_{\varphi}^{\infty}=\sup \left\{p: \varphi \in \Delta_{\infty}^{* p}\right\}, \\
\alpha_{\varphi}=\sup \left\{p: \varphi \in \Delta^{* p}\right\}, \\
\beta_{\varphi}^{0}=\inf \left\{q: \varphi \in \Delta_{0}^{q}\right\}, \quad \beta_{\varphi}^{\infty}=\inf \left\{q: \varphi \in \Delta_{\infty}^{q}\right\}, \\
\beta_{\varphi}=\inf \left\{q: \varphi \in \Delta^{q}\right\} .
\end{gathered}
$$

It is clear that $\alpha_{\varphi}=\min \left(\alpha_{\varphi}^{0}, \alpha_{\varphi}^{\infty}\right)$ and $\beta_{\varphi}=\max \left(\beta_{\varphi}^{0}, \beta_{\varphi}^{\infty}\right)$. We say that $\varphi$ satisfies conditions $\Delta_{2}^{0}, \Delta_{2}^{\infty}$, and $\Delta_{2}$, whenever

$$
\sup _{0<t<1} \frac{\varphi(2 t)}{\varphi(t)}<\infty, \quad \sup _{t>1} \frac{\varphi(2 t)}{\varphi(t)}<\infty, \quad \sup _{t>0} \frac{\varphi(2 t)}{\varphi(t)}<\infty,
$$


respectively. Let $\varphi_{1}$ and $\varphi_{2}$ be Orlicz functions. We call them equivalent at zero, equivalent at infinity, or just equivalent, if there is $D>0$ such that $\varphi_{1}\left(D^{-1} t\right) \leq \varphi_{2}(t) \leq \varphi_{1}(D t)$, for all $0<t<1, t>1$, or for all $t>0$, respectively. It is well known and easy to prove that Matuszewska-Orlicz indices of Orlicz functions are preserved by the corresponding equivalence relations (e.g., see [17]); that is, if $\varphi_{1}$ and $\varphi_{2}$ are equivalent (resp., equivalent at 0 and equivalent at $\infty$ ), then $\alpha_{\varphi_{1}}=\alpha_{\varphi_{2}}$ (resp., $\alpha_{\varphi_{1}}^{0}=\alpha_{\varphi_{2}}^{0}$ and $\alpha_{\varphi_{1}}^{\infty}=\alpha_{\varphi_{2}}^{\infty}$ ). Similar equalities hold for upper indices. Recall also that any upper index of $\varphi$ is finite if and only if $\varphi$ satisfies the corresponding condition $\Delta_{2}[17,18]$.

We also have that if the Orlicz functions $\varphi_{1}$ and $\varphi_{2}$ are equivalent (resp., equivalent at infinity and equivalent at zero), then $\Lambda_{\varphi_{1}, w}(0, \infty)=\Lambda_{\varphi_{2}, w}(0, \infty)$ (resp., $\Lambda_{\varphi_{1}, w}(0, a)=$ $\left.\Lambda_{\varphi_{2}, w}(0, a), a<\infty ; d\left(\varphi_{1}, w\right)=d\left(\varphi_{2}, w\right)\right)$ with equivalent norms.

The space $\Lambda_{\varphi, w}(0, \infty)\left(\operatorname{resp} ., \Lambda_{\varphi, w}(0, a)(a<\infty) ; d(\varphi, w)\right)$ is separable if and only if $\varphi \in \Delta_{2}$ and $W(\infty)=\infty$ (resp., $\varphi \in \Delta_{2}^{\infty} ; \varphi \in \Delta_{2}^{0}$ and $W(\infty)=\infty$ ), if $a=\infty$ (resp., $a<\infty$; $I=\mathbb{N})$. The separability is also equivalent to the case in which the space $\Lambda_{\varphi, w}(0, \infty)$ does not contain the isomorphic copy of $\ell_{\infty}[12,15,19]$.

Lemma 2. Let $1 \leq p, q<\infty$;

(1) ([20, Lemma 6], [21, Lemma 3], and [22]) An Orlicz function $\varphi \in \Delta^{* p}$ (resp., $\varphi \in \Delta_{\infty}^{* p} ; \varphi \in \Delta_{0}^{* p}$ ) if and only if there exists an Orlicz function $\psi$ equivalent (resp., equivalent at $\infty$; equivalent at 0$)$ to $\varphi$ such that $\psi\left(t^{1 / p}\right)$ is convex;

(2) ([20, Lemma 5], [21, Lemma 2], and [22]) An Orlicz function $\varphi \in \Delta^{q}$ (resp., $\varphi \in \Delta_{\infty}^{q} ; \varphi \in \Delta_{0}^{q}$ ) if and only if there exists an Orlicz function $\psi$ equivalent (resp., equivalent at $\infty$ and equivalent at 0 ) to $\varphi$ such that $\psi\left(t^{1 / q}\right)$ is concave.

We will use the following functions:

$$
\begin{gathered}
W(t)=\int_{0}^{t} w(s) d s \quad \text { for } t \in(0, a), \text { or } \\
W(m)=\sum_{i=1}^{m} w(i) \quad \text { for } m \in \mathbb{N} .
\end{gathered}
$$

Recall that the weight $w$ is regular whenever there exists $C>0$ such that $W(s) \leq C s w(s)$ for all $s \in I$. Since always $s w(s) \leq$ $W(s)$, the weight $w$ is regular if the functions $W(s)$ and $s w(s)$ are equivalent on $I$. Notice that for any regular weight $w$ on $I=(0, \infty)$ or $I=\mathbb{N}$ it holds that $W(\infty)=\infty$.

A Banach lattice is said to be $p$-convex (resp., $p$-concave) for some $1 \leq p<\infty$ if there is a constant $K>0$ such that

$$
\begin{gathered}
\left\|\left(\sum_{i=1}^{n}\left|x_{i}\right|^{p}\right)^{1 / p}\right\| \leq K\left(\sum_{i=1}^{n}\left\|x_{i}\right\|^{p}\right)^{1 / p} \\
\left(\operatorname{resp} \cdot\left(\sum_{i=1}^{n}\left\|x_{i}\right\|^{p}\right)^{1 / p} \leq K\left\|\left(\sum_{i=1}^{n}\left|x_{i}\right|^{p}\right)^{1 / p}\right\|\right)
\end{gathered}
$$

for every choice of vectors $x_{1}, \ldots, x_{n}$ in $X$. A Banach lattice is said to satisfy upper p-estimate (resp., lower p-estimate) if the definition of $p$-convexity (resp., $p$-concavity) holds true for any choice of disjointly supported elements $x_{1}, \ldots, x_{n}$ in $X$. It is known that, given $1 \leq p<\infty$, if $X$ is $p$-convex (resp., $p$ concave), then $X$ is $r$-convex (resp., $r$-concave) for $1 \leq r \leq p$ (resp., $r \geq p$ ) $[23,24]$.

Theorem 3 (see [25]). The space $\Lambda_{\varphi, w}(0, \infty)$ (resp., $\Lambda_{\varphi, w}(0, a)$ $(a<\infty) ; d(\varphi, w))$ has finite concavity if and only if $\varphi$ satisfies the $\Delta_{2}\left(\right.$ resp., $\left.\Delta_{2}^{\infty} ; \Delta_{2}^{0}\right)$ condition and the weight $w$ is regular.

Recall that a Banach lattice has the finite concavity if and only if it is of finite cotype [26].

Theorem 4 (see [19]). Let $1 \leq p \leq \infty$;

(1) [19, Theorem 7.18] For $I=(0, \infty)$, the following conditions are equivalent:

(i) $\ell^{p}$ (replaced by $c_{0}$ when $p=\infty$ ) is order isomorphic to a sublattice of $\Lambda_{\varphi, w}^{0}(0, \infty)$,

(ii) either $p \in\left[\alpha_{\varphi}^{0}, \beta_{\varphi}^{0}\right] \cup\left[\alpha_{\varphi}^{\infty}, \beta_{\varphi}^{\infty}\right]=\left[\alpha_{\varphi}, \beta_{\varphi}\right]$ or, for some $c>0, \int_{0}^{\infty} \varphi\left(c t^{-1 / p}\right) w(t) d t<\infty$;

(2) [19, Theorem 6.13] For $I=(0, a), a<\infty$, the following assertions are equivalent:

(i) $\ell^{p}$ (replaced by $c_{0}$ when $p=\infty$ ) is order isomorphic to a sublattice of $\Lambda_{\varphi, w}^{0}(0, a)$,

(ii) $p \in\left[\alpha_{\varphi}^{\infty}, \beta_{\varphi}^{\infty}\right]$,

(iii) $\ell^{p}$ (replaced by $c_{0}$ when $p=\infty$ ) is order isomorphic to a sublattice of $L_{\varphi}^{0}(0, a)$;

(3) [19, Theorem 7.8] For $I=\mathbb{N}$ the following statements are equivalent (where $c_{0}$ is meant in place of $\ell^{p}$ if $p=$ $\infty)$ :

(i) $\ell^{p}$ is order isomorphic to a sublattice of $d_{0}(w, \varphi)$,

(ii) $\ell^{p}$ is isomorphic to a subspace of $d_{0}(w, \varphi)$,

(iii) $p \in\left[\alpha_{\varphi}^{0}, \beta_{\varphi}^{0}\right]$,

(iv) $\ell^{p}$ is isomorphic to a subspace of $h_{\varphi}$.

\section{Main Results}

Theorem 5. If $1<p<\infty$ and $\varphi \in \Delta^{* p}$ (resp., $\varphi \in \Delta_{\infty}^{* p}$ and $\left.\varphi \in \Delta_{0}^{* p}\right)$, then $\Lambda_{\varphi, w}(0, \infty)$ (resp., $\Lambda_{\varphi, w}(0, a), a<\infty ; d(\varphi, w)$ ) is p-convex. If $\Lambda_{\varphi, w}(0, \infty)$ (resp., $\Lambda_{\varphi, w}(0, a), a<\infty$; $d(\varphi, w)$ ) is $p$-convex for some $1<p<\infty$, then $p \leq \alpha_{\varphi}$ (resp., $p \leq \alpha_{\varphi}^{\infty}$; $\left.p \leq \alpha_{\varphi}^{0}\right)$. 
Proof. (1) By Lemma 2 we assume that $\psi(t)=\varphi\left(t^{1 / p}\right)$ is convex. Then we have the equality $\|f\|_{\Lambda_{\psi, w}^{(p)}(I)}=\|f\|_{\varphi, w}$, where $\Lambda_{\psi, w}^{(p)}(I)$ is a $p$-convexification of $\Lambda_{\psi, w}(I)$. Indeed,

$$
\begin{aligned}
\|f\|_{\Lambda_{\psi, w}^{(p)}(I)} & =\left\||f|^{p}\right\|_{\psi, w}^{1 / p} \\
& =\inf \left\{\epsilon^{1 / p}>0: \int_{I} \psi\left(\frac{\left(|f|^{p}\right)^{*}}{\epsilon}\right) \leq 1\right\} \\
& =\inf \left\{\epsilon^{1 / p}>0: \int_{I} \varphi\left(\frac{f^{*}}{\epsilon^{1 / p}}\right) \leq 1\right\}=\|f\|_{\varphi, w} .
\end{aligned}
$$

Since $\psi$ is a convex Orlicz function, the space $\Lambda_{\psi, w}(I)$ is a Banach space, so its $p$-convexification $\Lambda_{\psi, w}^{(p)}(I)=\Lambda_{\varphi, w}(I)$ is p-convex [26].

We only show the second part in the case of $I=(0, \infty)$. If $p>\alpha_{\varphi}=\min \left(\alpha_{\varphi}^{0}, \alpha_{\varphi}^{\infty}\right)$, then, by Theorem 4(1), there is $\alpha_{\varphi} \leq r<p$ such that $\ell^{r}$ is an order copy in $\Lambda_{\varphi, w}(0, \infty)$, and thus the space cannot be $p$-convex.

Example 6. In general, the parallel statement on concavity in Theorem 5 does not hold. As shown by Pisier in [26, Example 1.f.19], the Lorentz space $d(p, w)$, where $w_{n}=n^{1 / p}-(n-1)^{1 / p}$ and $1<p<2$, is not 2-concave even though the function $\varphi(t)=t^{p}$ satisfies condition $\Delta_{2}$ and $w$ is regular.

Proposition 7. Let $1<p \leq 2$. If $\varphi \in \Delta^{* p}$ and $\varphi \in \Delta_{2}$ if $a=\infty$ (resp., $\varphi \in \Delta_{\infty}^{* p}$ and $\varphi \in \Delta_{2}^{\infty}$ in the case when $a<\infty$ ) and $w$ is regular, then $\Lambda_{\varphi, w}(0, a), 0<a \leq \infty$, has type $p$.

Proof. By Theorem 5 the space is $p$-convex, and thus in view of Theorem 3 and by applying the well-known fact [26, Proposition 1.f.3], the space has type $p$.

Rakov [27, Theorem 1] showed that if a Banach space has type $1<p \leq 2$, then it has $p$-Banach-Saks property. Hence we get the following.

Theorem 8. If a Banach space $X$ has type $p$ for some $1<p \leq$ 2 , then $X \in\left(B S_{p}\right)$. Consequently $p \leq \gamma(X)$.

Notice that for every separable r.i. space $E$ on $[0,1]$, it is shown in [28] that $\gamma(E) \leq 2$. Because every r.i. function space $X$ on $[0, \infty)$ contains the r.i. space $\widetilde{X}=\left\{f \chi_{[0,1]}: f \in X\right\}$ isometrically, we get the following.

Proposition 9 (see [29]). If $X$ is a separable r.i. space on $(0, a)$, $0<a \leq \infty$, then $\gamma(X) \leq 2$.

Recall that a Banach space $X$ is said to be $p$-smooth $(1 \leq$ $p \leq 2)$ if its modulus of smoothness $\rho_{X}(\epsilon)$ is majorized by the function $\epsilon^{p}$. That is, there is a constant $A>0$ such that, for all $\epsilon>0$,

$$
\rho_{X}(\epsilon):=\inf _{\{\|x\|=\|y\|=1\}} \frac{\|x+\epsilon y\|+\|x-\epsilon y\|-2}{2} \leq A \epsilon^{p} .
$$

Rakov [27, Theorem 1] showed that if $X$ is a $p$-smooth space, then it has the $\left(S_{p}\right)$ property. It is well known [26] that a Banach lattice is isomorphic to a $p$-smooth Banach space for some $1<p \leq 2$ if and only if it has the type $p$ and satisfies a lower $q$-estimate for some $1 \leq q<\infty$. Notice also that the finite concavity is equivalent to the lower $q$-estimate for some $q<\infty$ [26]. Then we obtain immediately the following corollary as a consequence of definition of lower indices and by Propositions 7 and 9 and Theorem 8 .

Corollary 10. Let $1<p \leq 2$. If $\varphi \in \Delta^{* p}$ and $\varphi \in \Delta_{2}$ if $a=\infty$ (resp., $\varphi \in \Delta_{\infty}^{* p}$ and $\varphi \in \Delta_{2}^{\infty}$ in the case when $a<\infty$ ) and $w$ is regular, then $\Lambda_{\varphi, w}(0, a), 0<a \leq \infty$, has the $S_{p}$-property.

Consequently, if $\varphi \in \Delta_{2}$ when $a=\infty$ (resp., $\varphi \in \Delta_{2}^{\infty}$ in the case when $a<\infty)$ and $w$ is regular, then

$$
\begin{array}{r}
\min \left(2, \alpha_{\varphi}\right) \leq \gamma\left(\Lambda_{\varphi, w}(0, \infty)\right), \\
\min \left(2, \alpha_{\varphi}^{\infty}\right) \leq \gamma\left(\Lambda_{\varphi, w}(0, a)\right), \\
a<\infty .
\end{array}
$$

The next corollary is a result of the facts that $\gamma\left(\ell^{\infty}\right)=1$ and that if $\varphi$ does not satisfy appropriate condition $\Delta_{2}$, then $\Lambda_{\varphi, w}(I)$ contains an isomorphic copy of $\ell^{\infty}$.

Corollary 11. If $\varphi \notin \Delta_{2}$ when $a=\infty, \varphi \notin \Delta_{2}^{\infty}$ in the case when $a<\infty$, and $\varphi \notin \Delta_{2}^{0}$ when $I=\mathbb{N}$, then

$$
\begin{array}{r}
\gamma\left(\Lambda_{\varphi, w}(0, \infty)\right)=1, \quad \gamma\left(\Lambda_{\varphi, w}(0, a)\right)=1, \\
a<\infty, \quad \gamma(d(\varphi, w))=1 .
\end{array}
$$

Corollary 12. An Orlicz-Lorentz space $\Lambda_{\varphi, w}^{0}(0, \infty)$ (resp., $\left.\Lambda_{\varphi, w}^{0}(0, a), a<\infty ; d^{0}(w, \varphi)\right)$ does not contain an isomorphic copy of $\ell^{1}$ if and only if $\alpha_{\varphi}>1$ (resp., $\alpha_{\varphi}^{\infty}>1 ; \alpha_{\varphi}^{0}>1$ ).

Proof. This hypothesis for sequence spaces is an immediate consequence of Theorem 4(3).

Let now $a<\infty$. From [19, Corollary 3.5(2), (3)], the space $\Lambda_{\varphi, w}^{0}(0, a)$ contains an isomorphic copy of $\ell^{1}$ if and only if either $\ell^{1}$ is an order isomorphic copy, that is, in view of Theorem $4(2), \alpha_{\varphi}^{\infty}=1$, or the sequence of functions $\min \left(t^{-1}, n\right)$ is bounded in $L_{\varphi}(0, a)$. However $\alpha_{\varphi}^{\infty}>1$ implies that there exists $p>1$ such that $\varphi(\beta u) \geq K \beta^{p} \varphi(u)$ for all $u, \beta \geq 1$ and some $K>0$. Hence for any constant $c>0$ and large enough $n \in \mathbb{N}$,

$$
\begin{aligned}
\int_{0}^{a} \varphi\left(c \min \left(t^{-1}, n\right)\right) d t & \geq \int_{0}^{1 / n} \varphi(c n) d t \\
& \geq \int_{0}^{1 / n} K c^{p} n^{p} \varphi(1) d t \\
& =K \varphi(c) n^{p-1} \longrightarrow \infty .
\end{aligned}
$$

Thus whenever $\alpha_{\varphi}^{\infty}>1$ the sequence $\min \left(t^{-1}, n\right)$ is not bounded, and the proof is done in case of finite interval. 
Assume that $a=\infty$. Again applying Corollary 3.5(2) in [19], we get dichotomy that $\ell^{1}$ is an isomorphic copy in $\Lambda_{\varphi, w}^{0}(0, \infty)$ if and only if either $\ell^{1}$ is an order isomorphic copy in $\Lambda_{\varphi, w}^{0}(0, \infty)$ or the sequence $\min \left(t^{-1} \chi_{(0,1)}(t), n\right)$ is bounded in $L_{\varphi}(0, \infty)$. Assuming now that $\alpha_{\varphi}>1$, we can show similarly as above that the second alternative cannot occur. Recall then that, by Theorem 4(1), $\ell^{1}$ is an order isomorphic copy in the space if and only if either $\alpha_{\varphi}=1$ or, for some $c>0, \int_{0}^{\infty} \varphi\left(c t^{-1}\right) w(t) d t<\infty$. We will exclude again the second possibility. In fact if $\alpha_{\varphi}>1$, then for some $p>1$ and $K>0$ and for all $\beta \geq 1$ and $u \geq 0, \varphi(\beta u) \geq K \beta^{p} \varphi(u)$. Therefore for every $c>0$ and some $t_{0}<1$ such that $w\left(t_{0}\right)>0$, we have

$$
\begin{aligned}
\int_{0}^{\infty} \varphi\left(c t^{-1}\right) w(t) d t & \geq \int_{0}^{t_{0}} \varphi\left(c t^{-1}\right) w\left(t_{0}\right) d t \\
& \geq K \varphi(c) w\left(t_{0}\right) \int_{0}^{t_{0}} t^{-p} d t=\infty
\end{aligned}
$$

and this completes the proof.

Corollary 13. Assume that $\Lambda_{\varphi, w}(0, a), 0<a \leq \infty$, does not contain an isomorphic copy of $\ell^{1}$, equivalently that $\alpha_{\varphi}>1$ in case $a=\infty$ and $\alpha_{\varphi}^{\infty}>1$ in case $a<\infty$. Then

$$
\begin{array}{r}
\gamma\left(\Lambda_{\varphi, w}(0, \infty)\right) \leq \min \left(2, \alpha_{\varphi}\right), \\
\gamma\left(\Lambda_{\varphi, w}(0, a)\right) \leq \min \left(2, \alpha_{\varphi}^{\infty}\right), \\
a<\infty .
\end{array}
$$

Proof. By Proposition 9 the Banach-Saks index of any r.i. space over the interval $(0, a)$ cannot be bigger than 2 . On the other hand, recall that $\Gamma\left(\ell_{p}\right)=[1, p]$ for $1<p<\infty$ and $\Gamma\left(c_{0}\right)=\Gamma\left(\ell_{1}\right)=[1, \infty]$. The space $\ell_{\infty}$ does not have $w B S$ property, so $\Gamma\left(\ell_{\infty}\right)=1$. Hence Theorem 4 shows that

$$
\begin{aligned}
& \gamma\left(\Lambda_{\varphi, w}(0, a)\right) \\
& \leq A \\
& :=\inf \left\{p>1: \ell^{p} \text { is an isomorphic copy in } \Lambda_{\varphi, w}(0, a)\right\},
\end{aligned}
$$

where $A \leq \alpha_{\varphi}$ when $a=\infty$ and $A \leq \alpha_{\varphi}^{\infty}$ if $a<\infty$.

Finally by Corollaries 10 and 11 we get the result on Banach-Saks index in the Orlicz-Lorentz spaces over the interval $(0, a), a \leq \infty$.

Theorem 14. Assume that $\Lambda_{\varphi, w}(0, a), 0<a \leq \infty$, does not contain an isomorphic copy of $\ell^{1}$ and equivalently that $\alpha_{\varphi}>1$ in case $a=\infty$ and $\alpha_{\varphi}^{\infty}>1$ in case $a<\infty$. Assume also that $\varphi \in \Delta_{2}$ when $a=\infty, \varphi \in \Delta_{2}^{\infty}$ when $a<\infty$, and $w$ is regular. Then

$$
\begin{array}{r}
\min \left(2, \alpha_{\varphi}\right)=\gamma\left(\Lambda_{\varphi, w}(0, \infty)\right), \\
\min \left(2, \alpha_{\varphi}^{\infty}\right)=\gamma\left(\Lambda_{\varphi, w}(0, a)\right), \\
a<\infty .
\end{array}
$$
ing.

Using the Bessaga-Pełczynski method, we get the follow-

Theorem 15. Let $1<p<\infty$. If an order continuous sequence space $X$ is p-convex, then $X$ has the $\left(S_{p}\right)$ property. Consequently $p \leq \gamma(X)$.

Proof. Since $X$ is order continuous, the standard unit vectors $\left\{e_{n}\right\}$ form a basis of $X$. Suppose that $\left\{x_{n}\right\}$ is a normalized weakly null sequence in $X$. By the Bessaga-Pełczyński method [5, Proposition 1.a.12], we may assume that $x_{n}=$ $y_{n}+z_{n}$ for all $n$, where $\left\{y_{n}\right\}$ is a block basic sequence of $\left\{e_{n}\right\}$ and $\left\|z_{n}\right\| \leq 1 / 2^{n}$ for all $n$. Then for real numbers $a_{1}, \ldots, a_{n}$ with $\sum_{j=1}^{n}\left|a_{j}\right|^{p} \leq 1$, the $p$-convexity of $X$ and disjointness of the supports of $y_{n}$ 's show that

$$
\begin{aligned}
\left\|\sum_{j=1}^{n} a_{j} x_{j}\right\| & \leq\left\|\sum_{j=1}^{n} a_{j} y_{j}\right\|+\sum_{j=1}^{n}\left|a_{j}\right|\left\|z_{j}\right\| \\
& \leq\left\|\left(\sum_{j=1}^{n}\left|a_{j} y_{j}\right|^{p}\right)^{1 / p}\right\|+\left(\sum_{j=1}^{n}\left|a_{j}\right|^{p}\right)^{1 / p}\left(\sum_{j=1}^{n} \frac{1}{2^{j q}}\right)^{1 / q} \\
& \leq 2 C\left(\sum_{j=1}^{n}\left|a_{j}\right|^{p}\right)^{1 / p}+\left(\sum_{j=1}^{n}\left|a_{j}\right|^{p}\right)^{1 / p}\left(\sum_{j=1}^{n} \frac{1}{2^{j q}}\right)^{1 / q} \\
& \leq(2 C+1)\left(\sum_{j=1}^{n}\left|a_{j}\right|^{p}\right)^{1 / p},
\end{aligned}
$$

where $C$ is the constant from the $p$-convexity of $X$ and $q$ is the conjugate of $p$ satisfying $1 / p+1 / q=1$. The proof is done.

As a consequence of the above Theorems 15 and 5 about $p$ convexity and Theorem 4 about $\ell^{p}$-copies, we get the BanachSaks index in sequence spaces as follows.

Theorem 16. Assume that $d(\varphi, w)$ does not contain an isomorphic copy of $\ell^{1}$ and equivalently that $\alpha_{\varphi}^{0}>1$. Assume also that $\varphi \in \Delta_{2}^{0}$ and $W(\infty)=\infty$. Then

$$
\gamma(d(\varphi, w))=\alpha_{\varphi}^{0} .
$$

Proof. By Theorems 5 and 15, $\alpha_{\varphi}^{0} \leq \gamma(d(\varphi, w))$ and

$$
\gamma(d(\varphi, w)) \leq \inf \left\{p>1: \ell^{p} \subset d(\varphi, w)\right\} .
$$

Since $\gamma\left(\ell_{p}\right)=p(1<p<\infty)$, Theorem 4 completes the proof.

We finish with a result on the weak Banach-Saks property. 
Theorem 17. A separable Orlicz-Lorentz space $\Lambda_{\varphi, w}(0, a), a \leq$ $\infty$, has the weak Banach-Saks property.

Proof. Let $\left\{x_{n}\right\} \subset \Lambda_{\varphi, w}(0, a)$ be weakly null and assume without loss of generality that $\left\|x_{n}\right\|=1, n \in \mathbb{N}$. Then by [29, Proposition 3.2] there exists a subsequence $\left\{x_{n}^{\prime}\right\}$ splitted into three subsequences

$$
x_{n}^{\prime}=y_{n}+z_{n}+d_{n}
$$

such that $\left\{y_{n}\right\},\left\{z_{n}\right\},\left\{d_{n}\right\} \subset \Lambda_{\varphi, w}(0, a)$ are bounded and

(a) $y_{1}^{*}=y_{n}^{*},\left\|y_{n}\right\| \leq 1$, and $y_{n} \rightarrow 0$ weakly;

(b) $z_{n} z_{m}=0$ for $n \neq m,\left\|z_{n}\right\| \leq 2, z_{n} \rightarrow 0$ in measure, and $z_{n} \rightarrow 0$ weakly;

(c) $\left\|d_{n}\right\| \rightarrow 0$.

We need to show that there is a subsequence of $\left\{x_{n}^{\prime}\right\}$ whose Cesàro means approach zero in norm. It is obvious that we can do it for the sequence $\left\{d_{n}\right\}$ satisfying (c). We will show this now for the sequence $\left\{y_{n}\right\}$. By [29, Proposition 4.3] there exists a subsequence $\left\{y_{n(k)}\right\} \subset\left\{y_{n}\right\}, x \in L_{1}(0, a)+L_{\infty}(0, a)$, such that

$$
\frac{1}{N} \sum_{k=1}^{N} y_{m(k)} \longrightarrow x
$$

in $L_{1}(0, a)+L_{\infty}(0, a)$ for all further subsequences $\left\{y_{m(k)}\right\} \subset$ $\left\{y_{n(k)}\right\}$ (we can assume that $y_{m(1)}=y_{1}$ ). Hence

$$
\frac{1}{N} \sum_{k=1}^{N} y_{m(k)} \longrightarrow x \quad \text { in measure on }(0, a) .
$$

(Recall that if $x_{n} \rightarrow 0$ in any r.i. space $X$, then $x_{n} \rightarrow 0$ in measure.) Since $y_{n(k)} \rightarrow 0$ weakly in $\Lambda_{\varphi, w}(0, a)$, we have, for any subsequence $(m(k))$ of $(n(k))$,

$$
\frac{1}{N} \sum_{k=1}^{N} y_{m(k)} \longrightarrow 0 \quad \text { weakly in } L_{1}(0, a)+L_{\infty}(0, a) .
$$

Applying now [29, Lemma 4.4] we get that

$$
\frac{1}{N} \sum_{k=1}^{N} y_{m(k)} \longrightarrow 0 \quad \text { in } L_{1}(0, a)+L_{\infty}(0, a)
$$

for all subsequences $\left\{y_{m(k)}\right\} \subset\left\{y_{n(k)}\right\}$. Let now $\left\{y_{m(k)}\right\}$ be an arbitrary subsequence of $\left\{y_{n(k)}\right\}$. Set

$$
a_{N}=\frac{1}{N} \sum_{k=1}^{N} y_{m(k)}
$$

By $y_{m(k)}^{*}=y_{1}^{*}$ and by subadditivity of the operator $t \mapsto \int_{0}^{t} f^{*}$, $f \in L^{0}(I)$, we get, for $t \geq 0$,

$$
\int_{0}^{t}\left(\frac{1}{N} \sum_{k=1}^{N} y_{m(k)}\right)^{*} \leq \frac{1}{N} \sum_{k=1}^{N} \int_{0}^{t} y_{m(k)}^{*}=\int_{0}^{t} y_{1}^{*}
$$

that is,

$$
a_{N} \prec \prec y_{1} \text {. }
$$

Since $a_{N} \rightarrow 0$ in measure and $a_{N} \prec \prec y_{1}$ for every $N \in \mathbb{N}$, by [29, Proposition 4.2],

$$
\frac{1}{N} \sum_{k=1}^{N} y_{m(k)} \longrightarrow 0
$$

in $\Lambda_{\varphi, w}(0, a)$, which finishes the case (a).

In the case (b), by [19, Theorem 5.5], there exists a subsequence $\left\{z_{n(k)}\right\} \subset\left\{z_{n}\right\}$ such that any further subsequence $\left\{z_{m(k)}\right\}$ of $\left\{z_{n(k)}\right\}$ is equivalent to the unit vector basis in some Orlicz sequence space $\ell_{\psi}^{0}$. Thus $z_{n(k)} \rightarrow 0$ weakly in $\ell_{\psi}^{0}$, and by $w B S$-property of $\ell_{\psi}^{0}$ (see, e.g., [27]), there is a subsequence $\left\{z_{k}^{\prime}\right\} \subset\left\{z_{n(k)}\right\}$ such that $(1 / n) \sum_{k=1}^{N} z_{k}^{\prime} \rightarrow 0$ in $\ell_{\psi}^{0}$ and thus in $\Lambda_{\varphi, w}(0, a)$. This completes case (b) and proves the theorem.

A Banach space $X$ is said to have the (resp., weakly) alternative signs Banach-Saks property (ABS) (resp., (wABS)) if, for every bounded (resp., weakly null) sequence $\left(x_{n}\right)_{n=1}^{\infty}$, there is a subsequence $\left(y_{n}\right)$ and a sequence $\left(\epsilon_{n}\right)$ of signs such that the Cesàro averages are norm convergent to zero. That is,

$$
\lim _{n \rightarrow \infty} \frac{1}{n}\left\|\sum_{i=1}^{n} \epsilon_{i} y_{i}\right\|=0 \text {. }
$$

It is shown by Rosenthal $[30,31]$ that if $X$ has the (ABS), then $X$ has the weak Banach-Saks property. In case that $X$ does not contain $\ell_{1}$, then (ABS) is equivalent to the weak Banach-Saks property [31]. It is clear that $\ell_{1}$ does not have the (ABS) and has the weak Banach-Saks property. Hence a Banach space $X$ has the (ABS) if and only if $X$ has the weak Banach-Saks property and does not contain $\ell_{1}$. The notion of (wABS) was introduced in [32] and it was shown that (wABS) is equivalent to (wBS).

Corollary 18. Let $I=(0, a)$ for some $0<a \leq \infty$. Then the following are equivalent:

(1) Orlicz-Lorentz space $\Lambda_{\varphi, w}(I)$ has the Banach-Saks property (BS);

(2) Orlicz-Lorentz space $\Lambda_{\varphi, w}(I)$ is reflexive;

(3) Orlicz-Lorentz space $\Lambda_{\varphi, w}(I)$ does not contain isomorphic copies of $c_{0}$ or $\ell_{1}$;

(4) Orlicz-Lorentz space $\Lambda_{\varphi, w}(I)$ is separable and does not contain an isomorphic copy of $\ell_{1}$;

(5) Orlicz-Lorentz space $\Lambda_{\varphi, w}(I)$ has the weak BanachSaks property ( $w B S)$ and does not contain an isomorphic copy of $\ell_{1}$;

(6) Orlicz-Lorentz space $\Lambda_{\varphi, w}(I)$ has the alternative signs Banach-Saks property (ABS).

Proof. Recall that a Banach lattice is reflexive if and only if it does not contain isomorphic copies of $c_{0}$ or $\ell_{1}$ 
[26, Theorem 1.c.5]. Hence we get $(2) \Leftrightarrow(3)$. Recall also that a Banach lattice $X$ is a KB space if and only if $X$ does not contain an isomorphic copy of $c_{0}$ [33, Theorem 14.12]. That is, if $X$ is a Köthe function space, then it is equivalent to the case in which $X$ is order continuous and satisfies the Fatou property. Since an Orlicz-Lorentz space satisfies the Fatou property and the order continuity is equivalent to the separability of the OrliczLorentz space, $(3) \Leftrightarrow$ (4) follows.

Since a nonseparable Orlicz-Lorentz space contains $\ell_{\infty}$ and it does not have the weak Banach-Saks property, Theorem 17 shows that $(4) \Leftrightarrow(5)$. The preceding argument of the corollary shows that (5) $\Leftrightarrow(6)$. Since (6) implies both (5) and (2), (6) implies (1). The proof is done.

\section{Conflict of Interests}

The authors declare that there is no conflict of interests regarding the publication of this paper.

\section{Acknowledgment}

Han Ju Lee was supported by Basic Science Research Program through the National Research Foundation of Korea(NRF) funded by the Ministry of Education, Science and Technology (NRF-2012R1A1A1006869).

\section{References}

[1] P. K. Lin, Köthe-Bochner Function Spaces, Birkhäuser, Boston, Mass, USA, 2004.

[2] J. Schreier, "Ein Gegenbeispiel zur theorie der schwachen konvergentz," Studia Mathematica, vol. 2, pp. 58-62, 1930.

[3] A. Baernstein, II, "On reflexivity and summability," Studia Mathematica, vol. 42, pp. 91-94, 1972.

[4] W. B. Johnson, "On quotients of $L_{p}$ which are quotients of $l_{p}$," Compositio Mathematica, vol. 34, no. 1, pp. 69-89, 1977.

[5] J. Lindenstrauss and L. Tzafriri, Classical Banach Spaces I, Springer, Berlin, Germany, 1977.

[6] A. Kamínska and H. J. Lee, "Banach-Saks Properties of Musielak-Orlicz and Nakano sequence spaces," Proceedings of the American Mathematical Society, vol. 142, no. 2, pp. 547-558, 2014.

[7] H. Knaust and E. Odell, "Weakly null sequences with upper $l_{p^{-}}$ estimates," in Functional Analysis, vol. 1470 of Lecture Notes in Mathematics, pp. 85-107, Springer, Berlin, Germany, 1991.

[8] S. A. Argyros, G. Godefroy, and H. P. Rosenthal, "Descriptive set theory and Banach spaces," in Handbook of the Geometry of Banach Spaces, vol. 2, pp. 1007-1069, North-Holland, Amsterdam, The Netherlands, 2003.

[9] P. Cembranos, "The hereditary Dunford-Pettis property on $C(K, E)$," Illinois Journal of Mathematics, vol. 31, no. 3, pp. 365373, 1987.

[10] H. Knaust, "Orlicz sequence spaces of Banach-Saks type," Archiv der Mathematik, vol. 59, no. 6, pp. 562-565, 1992.

[11] S. A. Rakov, "The Banach-Saks property for a Banach space," Matematicheskie Zametki, vol. 26, no. 6, pp. 823-834, 1979.
[12] J. Cerdà, H. Hudzik, A. Kamińska, and M. Mastyło, “Geometric properties of symmetric spaces with applications to OrliczLorentz spaces," Positivity, vol. 2, no. 4, pp. 311-337, 1998.

[13] H. Hudzik, A. Kamińska, and M. Mastyło, "Geometric properties of some Calderón-Lozanovskil spaces and Orlicz-Lorentz spaces," Houston Journal of Mathematics, vol. 22, no. 3, pp. 639663, 1996.

[14] H. Hudzik, A. Kamińska, and M. Mastyło, "On the dual of Orlicz-Lorentz space," Proceedings of the American Mathematical Society, vol. 130, no. 6, pp. 1645-1654, 2002.

[15] A. Kamińska, "Some remarks on Orlicz-Lorentz spaces," Mathematische Nachrichten, vol. 147, pp. 29-38, 1990.

[16] A. Kamińska and L. Maligranda, "Order convexity and concavity of Lorentz spaces $\Lambda_{p, w}, 0<p<\infty$," Studia Mathematica, vol. 160, no. 3, pp. 267-286, 2004.

[17] A. Kamińska, L. Maligranda, and L. E. Persson, "Indices and regularizations of measurable functions," in Function Spaces, vol. 213 of Lecture Notes in Pure and Applied Mathematics, pp. 231-246, Marcel Dekker, New York, NY, USA, 2000.

[18] W. Matuszewska and W. Orlicz, "On certain properties of $\phi$ functions," Bulletin de l'Académie Polonaise des Sciences, vol. 8 , pp. 439-443, 1960.

[19] A. Kamińska and Y. Raynaud, "Isomorphic copies in the lattice $E^{(*)}$ with applications to Orlicz-Lorentz spaces," Journal of Functional Analysis, vol. 257, no. 1, pp. 271-331, 2009.

[20] E. Katirtzoglou, "Type and cotype of Musielak-Orlicz sequence spaces," Journal of Mathematical Analysis and Applications, vol. 226, no. 2, pp. 431-455, 1998.

[21] A. Kamińska and B. Turett, "Type and cotype in MusielakOrlicz spaces," in Geometry of Banach Spaces, vol. 158 of London Mathematical Society Lecture Note Series, pp. 165-180, Cambridge Univeristy Press, Cambridge, UK, 1990.

[22] A. Kamińska, "Indices, convexity and concavity in MusielakOrlicz spaces," Functiones et Approximatio Commentarii Mathematici, vol. 26, pp. 67-84, 1998.

[23] B. Cuartero and M. A. Triana, “( $p, q)$-convexity in quasi-Banach lattices and applications," Studia Mathematica, vol. 84, no. 2, pp. 113-124, 1986.

[24] N. J. Kalton, "Convexity conditions for nonlocally convex lattices," Glasgow Mathematical Journal, vol. 25, no. 2, pp. 141152, 1984.

[25] A. Kamińska, L. Maligranda, and L. E. Persson, "Indices, convexity and concavity of Calderón-Lozanovskii spaces," Mathematica Scandinavica, vol. 92, no. 1, pp. 141-160, 2003.

[26] J. Lindenstrauss and L. Tzafriri, Classical Banach Spaces II, Springer, Berlin, Germany, 1979.

[27] S. A. Rakov, "The Banach-Saks exponent of some Banach spaces of sequences," Matematicheskie Zametki, vol. 32, no. 5, pp. 613625, 1982.

[28] E. M. Semënov and F. A. Sukochev, "The Banach-Saks index," Sbornik Matematicheskǐ, vol. 195, no. 2, pp. 263-285, 2004.

[29] P. G. Dodds, E. M. Semenov, and F. A. Sukochev, "The BanachSaks property in rearrangement invariant spaces," Studia Mathematica, vol. 162, no. 3, pp. 263-294, 2004.

[30] H. P. Rosenthal, "Weakly independent sequences and the Banach-Saks property," in Proceedings of the Durham Symposium on the Relations between Infinite Dimensional Convexity, p. 26, Durham, UK, July 1975. 
[31] B. Beauzamy, "Banach-Saks properties and spreading models," Mathematica Scandinavica, vol. 44, no. 2, pp. 357-384, 1979.

[32] K. Cho and C. Lee, "Alternative signs averaging properties in Banach spaces," Journal of Applied Mathematics and Computing, vol. 16, pp. 497-507, 2004.

[33] C. A. Aliprantis and O. Burkinshaw, Positive Operators, vol. 119, Academic Press, Orlando, Fla, USA, 1985. 


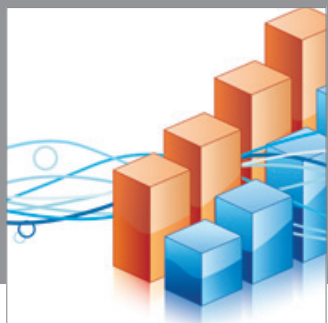

Advances in

Operations Research

mansans

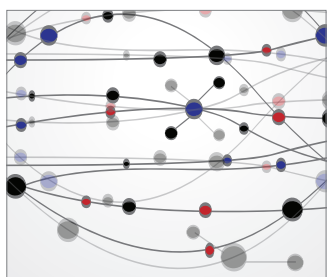

The Scientific World Journal
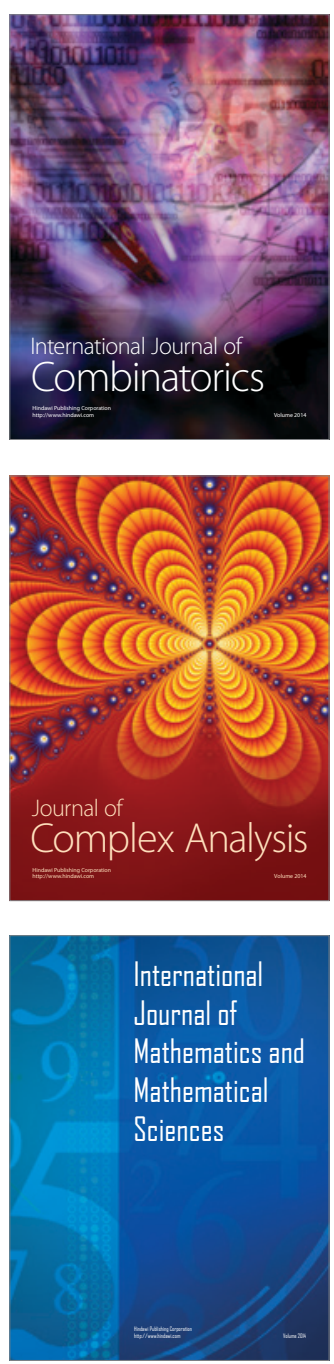
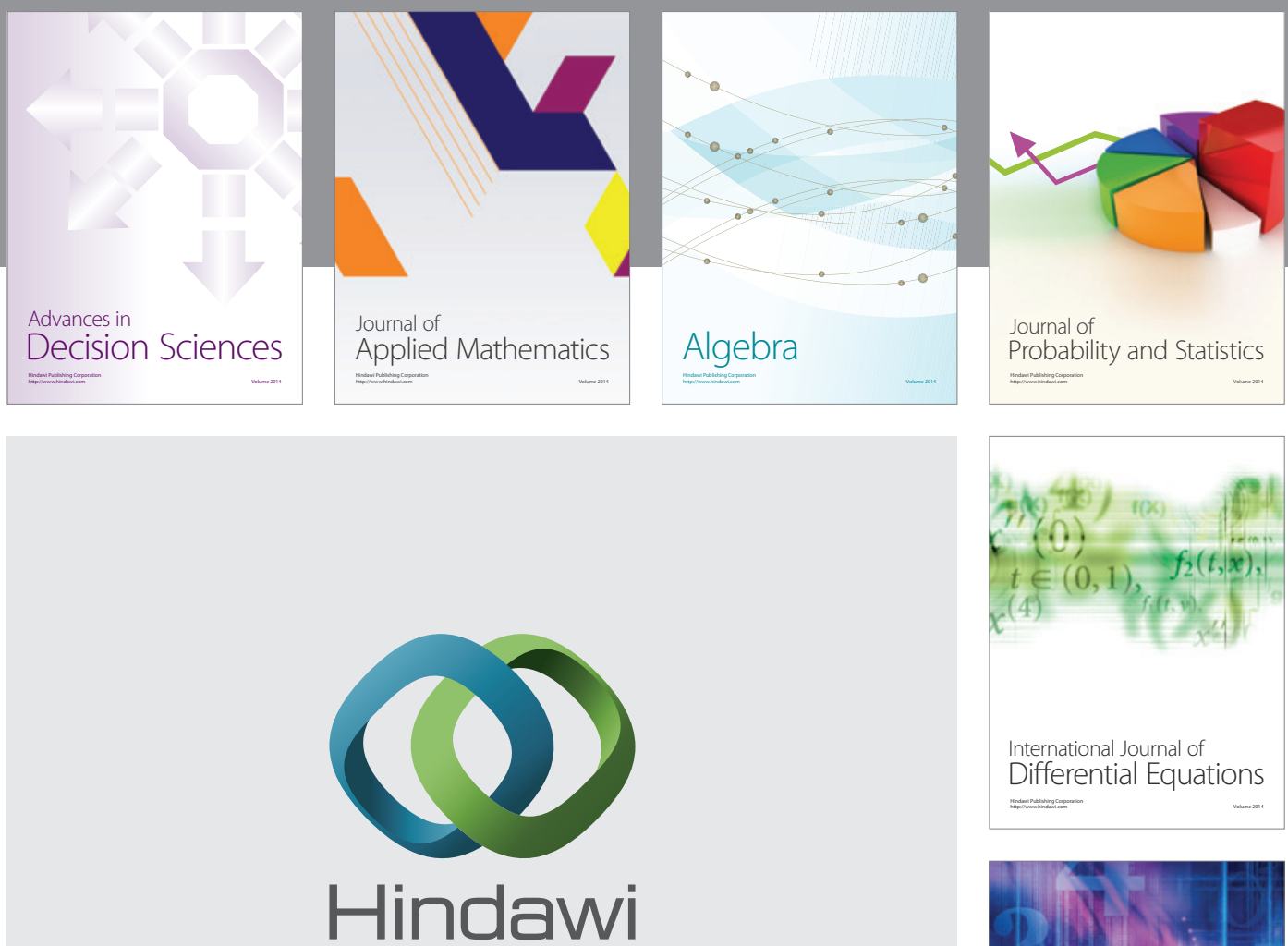

Submit your manuscripts at http://www.hindawi.com
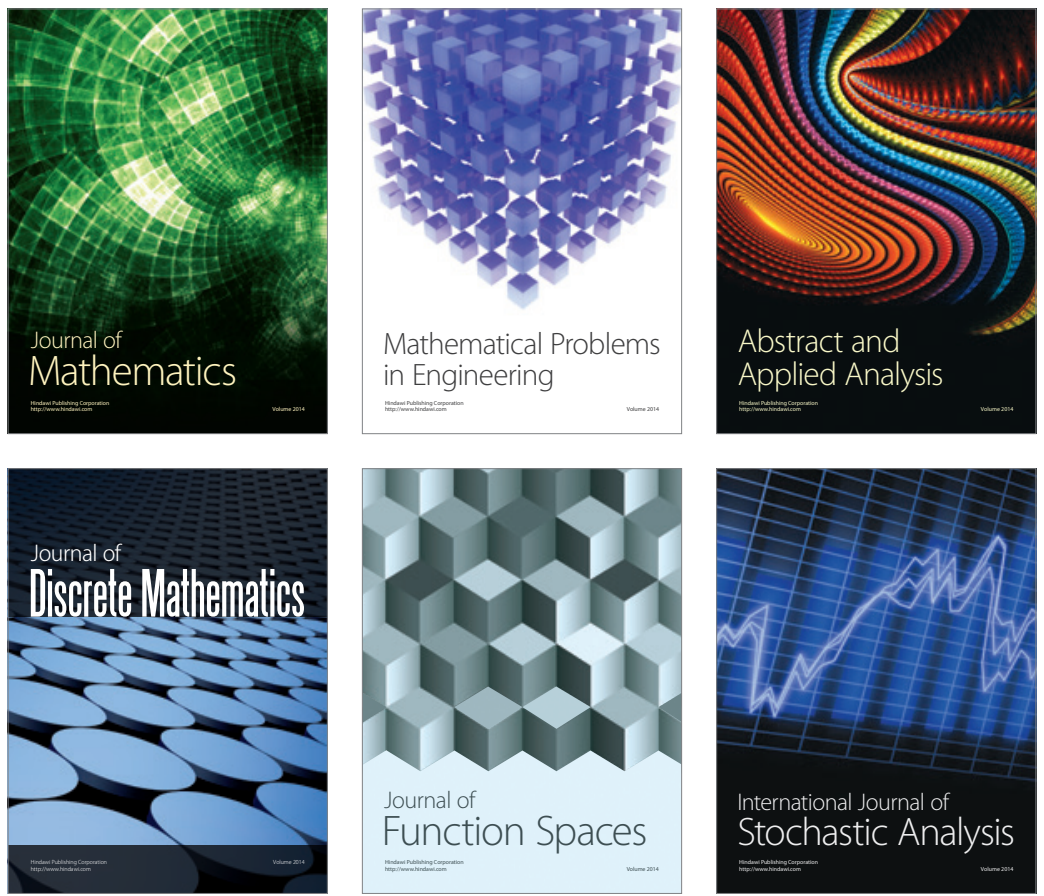

Journal of

Function Spaces

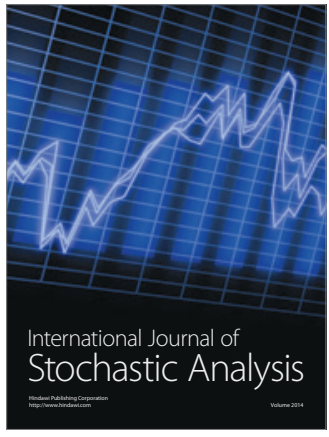

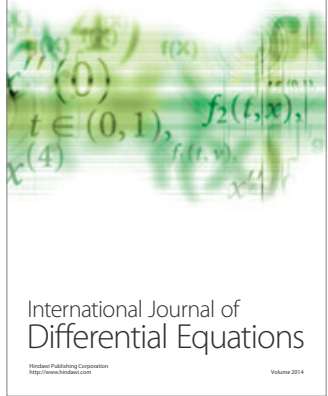
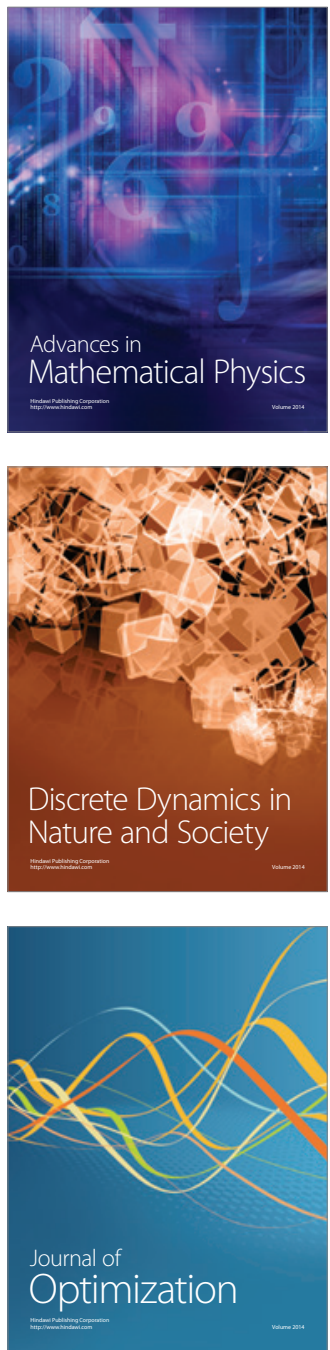